\title{
MODEL ELLIPTIC BOUNDARY-VALUE PROBLEMS FOR PSEUDODIFFERENTIAL OPERATORS IN CANONICAL NONSMOOTH DOMAINS
}

\author{
V. B. Vasilyev
}

UDC $517.95+517.983$

\begin{abstract}
We consider a simplest elliptic pseudodifferential equation in a multi-dimensional cone (multi-dimensional angle) and describe all possible structures of its solutions related to the wave factorization of the elliptic symbol. Depending on the index of wave factorization, we consider various statements of well-posed boundary-value problems. The existence of solutions is studied in Sobolev-Slobodetskii spaces.
\end{abstract}

\section{Introduction}

This work pertains to the next, "multi-dimensional," stage in the development of the theory of boundary-value problems for elliptic pseudodifferential operators on manifolds whose boundary may contain various types of singularities. This program was proposed by the author in the late 1980s to early 1990s and was partially realized in his works of that period: at least a fairly complete understanding of the two-dimensional situation was reached (a review and the results of these papers can be found $[4,5]$ ). The principal difference of the author's approach from previous works lies in a systematic utilization of the concept of wave factorization of the elliptic symbol. Roughly speaking, we are dealing with a multi-dimensional version of the Wiener-Hopf method or a multi-dimensional analogue of the classical Riemann boundary-value problem $[1,2]$. A few such analogues had been proposed (V. A. Kakichev, V. S. Vladimirov, et al.), but none of those are suitable for the construction of a theory of pseudodifferential equations on manifolds with nonsmooth boundaries. The author's main idea is that the construction of such a theory should be based on merely two fundamental principles:

- the local principle (adopted by I. B. Simonenko with regard to multi-dimensional singular integral equations), otherwise called "the principle of frozen coefficients" (used by J. Schauder for partial differential equations);

- the factorization principle, the efficiency of which has been convincingly demonstrated in the works of M. I. Vishik and G. I. Eskin and which has been supplemented by the author's introduction of wave factorization at a singular boundary point.

The simplest domain with a nonsmooth boundary is a cone in $m$-dimensional space. Starting with the classical work of V. A. Kondratiev, such a cone has been treated as the direct product of the base and the semi-axis, with further application of the Mellin transformation in one variable and the reduction of the original boundary-value problem in the cone to the corresponding boundary-value problem with a parameter, now in a domain with a smooth boundary (the base of the cone). The author has allowed himself to question whether this approach is reasonable, since the result of this procedure is not a cone but a cylinder, which is a completely different geometric figure. Further, what considerations should be used for the statement of one or another problem? This is where the divergence starts. First, the author is of the opinion that the cone should not be subject to "splitting," since it represents one of the many canonical singularities (the initial model singularity for the points of a smooth boundary was the half-space $\mathbb{R}_{+}^{m}$, and in this case the inversion of the model elliptic pseudodifferential operator can be effectively based on the theory of the classical Riemann boundary-value problem and the Cauchy integral). Second, in the

Translated from Trudy Seminara imeni I. G. Petrovskogo, No. 31, pp. 22-37, 2016. 
framework of the Vishik-Eskin theory, everything is explained in such a simple and natural way that one inevitably lands at trying to generalize all this to the multi-dimensional case.

Many analysts started their investigations directly with Lipschitz domains, more precisely, manifolds whose boundary can be locally represented as the graph of a Lipschitz continuous function. However, at the Equadiff-2013 conference in Prague, V. G. Maz'ya managed to persuade the author (in particular, by drawing a very simple picture) that there are many cones that cannot be described by such graphs, not to mention exotic situations, when one has to deal with "bunches" of different singularities (see [6]).

Some preliminary and additional results obtained by the author in relation to the present work can be found in [7-11].

\section{Model Operators and Canonical Domains}

2.1. Pseudodifferential Operators. These operators are locally defined by the formula

$$
u(x) \mapsto \int_{\mathbb{R}^{m}} \int_{\mathbb{R}^{m}} A(\cdot, \xi) u(y) e^{i(x-y) \cdot \xi} d \xi d y,
$$

provided that $D$ is a smooth compact manifold, since in this case one can use the principle of frozen coefficients, which is also called "the local principle." For manifolds with a smooth boundary, other local formulas are required to define an operator $A$ : namely, at the interior points of the manifold $D$, one utilizes the first formula, but at the boundary points, another one:

$$
u(x) \mapsto \int_{\mathbb{R}_{+}^{m}} \int_{\mathbb{R}^{m}} A(\cdot, \xi) u(y) e^{i(x-y) \cdot \xi} d \xi d y,
$$

where $\mathbb{R}_{+}^{m}=\left\{x \in \mathbb{R}^{m}: x=\left(x_{1}, \ldots, x_{m}\right), x_{m}>0\right\}$ is a half-space.

For the inversion of the latter operator with the symbol $A(\cdot, \xi)$ independent of the spatial variable $x$, one can use the theory of the classical Riemann boundary-value problem $[1,2]$ for the upper and the lower complex half-planes with the parameter $\xi^{\prime}$. A systematic realization of this procedure can be found in the works of M. I. Vishik and G. I. Eskin (see [3]). However, if the boundary $\partial D$ contains a conical point, this approach is inapplicable.

A conical point on the boundary is a point with a neighborhood diffeomorphic to the cone

$$
C_{+}^{a}=\left\{x \in \mathbb{R}^{m}: x_{m}>a\left|x^{\prime}\right|, x^{\prime}=\left(x_{1}, \ldots, x_{m-1}\right), a>0\right\},
$$

which gives rise to the following local definition of a pseudodifferential operator in a neighborhood of a conical point:

$$
u(x) \mapsto \int_{C_{+}^{a}} \int_{\mathbb{R}^{m}} A(\cdot, \xi) u(y) e^{i(x-y) \cdot \xi} d \xi d y .
$$

2.2. Sobolev-Slobodetskii Spaces. In the multi-dimensional space $\mathbb{R}^{n+m}$, we introduce the set $K_{n} \equiv$ $\mathbb{R}^{n} \times C_{+}^{a}$ and consider the equation

$$
(A u)(x)=f(x), \quad x \in K_{n},
$$

where $A$ is a pseudodifferential operator with the corresponding model symbol satisfying the following condition: $\exists c_{1}, c_{2}>0$ such that

$$
c_{1} \leq\left|A(\xi)(1+|\xi|)^{-\alpha}\right| \leq c_{2}, \quad \xi \in \mathbb{R}^{n+m} .
$$

The real $\alpha \in \mathbb{R}$ is called the order of the pseudodifferential operator $A$.

We consider such operators in the Sobolev-Slobodetskii space $H^{s}\left(\mathbb{R}^{n+m}\right)$ with the norm

$$
\|u\|_{s}^{2}=\int_{\mathbb{R}^{n+m}}|\tilde{u}(\xi)|^{2}(1+|\xi|)^{2 s} d \xi .
$$


It is well known (and can be easily proved) that $A$ is a bounded linear operator from $H^{s}\left(\mathbb{R}^{n+m}\right)$ to $H^{s-\alpha}\left(\mathbb{R}^{n+m}\right)[3]$.

\section{Wave Factorization and Existence of Solutions}

Denote by $\stackrel{*}{a}_{+}^{a}$ the cone conjugate to $C_{+}^{a}$ :

$$
C_{+}^{*}=\left\{x \in \mathbb{R}^{m}: x=\left(x^{\prime}, x_{m}\right), a x_{m}>\left|x^{\prime}\right|\right\},
$$

and let $C_{-}^{a} \equiv-C_{+}^{a}$. By $T\left(C_{+}^{a}\right)$ we denote the radial tubular region over the cone $C_{+}^{a}$, i.e., the region of the form $\mathbb{R}^{m}+i C_{+}^{a}$ in the multi-dimensional complex space $\mathbb{C}^{m}$. The symbol tilde " " is used to denote the Fourier transform of a function, as well as the Fourier image of a space, respectively: $\tilde{u}_{+}, u_{+}$and $\tilde{H}, H$. The variable $\xi \in \mathbb{R}^{n+m}$ is represented in the form $\xi=\left(\xi^{\prime \prime}, \zeta\right)$, where $\xi^{\prime \prime} \in \mathbb{R}^{n}, \zeta \in \mathbb{R}^{m}, \zeta=\left(\xi^{\prime}, \xi_{m}\right)$.

To describe the situation with the existence of solutions, we introduce the following notion.

Definition 1. For a symbol $A(\xi)$, its wave factorization with respect to the cone $K_{n}$ is its representation of the form

$$
A(\xi)=A_{\neq}(\xi) A_{=}(\xi)
$$

where $A_{\neq}(\xi)$ and $A_{=}(\xi)$ satisfy the following conditions:

(1) $A_{\neq}(\xi), A_{=}(\xi)$ are defined for all $\xi \in \mathbb{R}^{n+m}$, except, possibly, the points $\left\{\xi \in \mathbb{R}^{n+m}:\left|\xi^{\prime}\right|^{2}=a^{2} \xi_{m}^{2}\right\}$;

(2) $A_{\neq}(\xi), A_{=}(\xi)$ can be analytically extended to the radial tubular regions $T\left(\stackrel{*}{C_{+}^{a}}\right), T\left({ }^{*} C_{-}^{a}\right)$ for almost all $\xi^{\prime \prime} \in \mathbb{R}^{n}$ such that, respectively,

$$
\left|A_{\neq}^{ \pm 1}\left(\xi^{\prime \prime}, \zeta+i \tau\right)\right| \leq c_{1}(1+|\xi|+|\tau|)^{ \pm \varkappa}, \quad\left|A_{=}^{ \pm 1}\left(\xi^{\prime \prime}, \zeta-i \tau\right)\right| \leq c_{2}(1+|\xi|+|\tau|)^{ \pm(\alpha-\varkappa)}, \forall \tau \in C_{+}^{*} .
$$

The exponent $\varkappa \in \mathbb{R}$ is called the wave factorization index.

The class of such symbols is sufficiently wide: relevant examples can be found, for instance, in $[4,7]$.

Note that wide classes of analytic functions in tubular regions over cones have been examined in detail by V. S. Vladimirov $[17,18]$.

We introduce the space $H^{s}\left(K_{n}\right)$ that consists of generalized functions in $H^{s}\left(\mathbb{R}^{n+m}\right)$ with support in $\bar{K}_{n}$. The right-hand side of the equation is chosen from the space of generalized functions in $H_{0}^{s-\alpha}\left(K_{n}\right)$ that can be extended to $H^{s-\alpha}\left(\mathbb{R}^{n+m}\right)$. The norm in $H_{0}^{s}\left(K_{n}\right)$ is defined as

$$
\|f\|_{s-\alpha}^{+}=\inf \|l f\|_{s-\alpha},
$$

where the infimum is taken over all possible extensions lf.

In what follows, it is always assumed that the symbol $A(\xi)$ allows for a wave factorization with respect to $K_{n}$ and its wave factorization index is $\varkappa$.

3.1. The Case of a Unique Solution. Let $\varkappa-s=\delta,|\delta|<1 / 2$. We introduce an integral operator $G_{n, m}$ by

$$
\left(G_{n, m} u\right)(\xi)=\frac{a \Gamma(m / 2)}{2 \pi^{(m+2) / 2}} \lim _{\tau \rightarrow 0+} \int_{\mathbb{R}^{m}} \frac{u\left(\xi^{\prime \prime}, \eta^{\prime}, \eta_{m}\right) d \eta^{\prime} d \eta_{m}}{\left(\left|\xi^{\prime}-\eta^{\prime}\right|^{2}-a^{2}\left(\xi_{m}-\eta_{m}+i \tau\right)^{2}\right)^{m / 2}},
$$

where $\Gamma$ is the Euler gamma-function.

The structure of the operator $G_{n, m}$ is very simple. It is the Fourier transform of the multiplicator generated by the characteristic function of the cone $K_{n}$. Since this function is nonintegrable, one has to introduce an "additional coefficient" of the type $e^{i \tau}$, which, in the Fourier transforms, results in the convolution with the Bochner kernel $[17,18]$ and the exit to a multi-dimensional complex space.

Such operators are constructed in explicit form in $[14,15]$ for $n=0$, as well as $m=2$. In our case, the situation is much more difficult.

Lemma 1. For $|\delta|<1 / 2$, the operator $G_{n, m}$ is a bounded linear operator $\tilde{H}^{\delta}\left(\mathbb{R}^{n+m}\right) \rightarrow \tilde{H}^{\delta}\left(\mathbb{R}^{n+m}\right)$. 
For the most part, the proof follows the arguments used in $[3,4,14]$ and is omitted.

Theorem 1. Let $\varkappa-s=\delta,|\delta|<1 / 2$. Then, equation (1) has a unique solution in $H^{s}\left(K_{n}\right)$ for any right-hand side $f \in H_{0}^{s-\alpha}\left(K_{n}\right)$. This solution has the form (in terms of Fourier transforms)

$$
\tilde{u}_{+}(\xi)=A_{\neq}^{-1}(\xi) G_{n, m} A_{=}^{-1} \widetilde{l f} .
$$

Proof. Let us extend $f$ to the whole of $\mathbb{R}^{n+m}$ and denote the extension by $l f$. Setting

$$
u_{-}(x)=\left(A u_{+}\right)(x)-l f(x),
$$

we rewrite equation (1) as

$$
\left(A u_{+}\right)(x)+u_{-}(x)=l f(x) .
$$

Now passing to Fourier transforms and using the wave factorization, we can write the original equation as follows:

$$
A_{\neq}(\xi) \tilde{u}_{+}(\xi)+A_{=}^{-1}(\xi) \tilde{u}_{-}(\xi)=A_{=}^{-1}(\xi) \widetilde{l f}(\xi) .
$$

Note that $A_{=}^{-1}(\xi) \widetilde{l f}(\xi)$ belongs to $\tilde{H}^{s-\varkappa}\left(\mathbb{R}^{n+m}\right)$ and $|s-\varkappa|<1 / 2$ by assumption. In such spaces, the operator $A_{=}^{-1}$ is bounded, and therefore, according to the theory of the multi-dimensional Riemann problem [14], the right-hand side $A_{=}^{-1}(\xi) \widetilde{l f}(\xi)$ can be uniquely represented as the sum

$$
\begin{gathered}
A_{=}^{-1}(\xi) \widetilde{l f}(\xi)=f_{+}(\xi)+f_{-}(\xi), \\
f_{+}(\xi)=G_{n, m} A_{=}^{-1}(\xi) \widetilde{l f}(\xi), \quad f_{-}(\xi)=\left(I-G_{n, m}\right) A_{=}^{-1}(\xi) \widetilde{l f}(\xi) .
\end{gathered}
$$

Let us rewrite (2) in the form

$$
A_{\neq}(\xi) \tilde{u}_{+}(\xi)-f_{+}(\xi)=f_{-}(\xi)-A_{=}^{-1}(\xi) \tilde{u}_{-}(\xi),
$$

where $f_{+} \in \tilde{H}^{-\delta}\left(K_{n}\right), f_{-} \in \tilde{H}^{-\delta}\left(\mathbb{R}^{n+m} \backslash K_{n}\right)$. The right- and left-hand sides of the last relation belong to $\tilde{H}^{-\delta}\left(K_{n}\right)$ and $\tilde{H}^{-\delta}\left(\mathbb{R}^{n+m} \backslash K_{n}\right)$, respectively, and are therefore annihilated, since $|\delta|<1 / 2$. Thus,

$$
\tilde{u}_{+}(\xi)=A_{\neq}^{-1}(\xi) G_{n, m} A_{=}^{-1} \widetilde{l f}
$$

which completes the proof.

3.2. Structure of the General Solution. Here, we consider equation (1) for $\varkappa-s=k+\delta, k \in \mathbb{N}$, $|\delta|<1 / 2$. Proceeding as above up to formula (2), taking into account that $A_{=}^{-1}(\xi) \widetilde{l f}(\xi)$ belongs to $\tilde{H}^{s-\varkappa}\left(\mathbb{R}^{n+m}\right)$, and choosing a polynomial $Q(\xi)$ such that

$$
|Q(\xi)| \sim(1+|\xi|)^{k}
$$

we find that the function $Q^{-1}(\xi) A_{=}^{-1}(\xi) \widetilde{l f}(\xi)$ belongs to $\tilde{H}^{-\delta}\left(\mathbb{R}^{n+m}\right)$.

Theorem 2. In terms of Fourier transforms, the general solution of equation (1) has the form

$$
\tilde{u}_{+}(\xi)=A_{\neq}^{-1}(\xi) Q(\xi) G_{n, m} Q^{-1}(\xi) A_{=}^{-1}(\xi) \widetilde{l f}(\xi)+A_{\neq}^{-1}(\xi) V_{-a} F\left(\sum_{j=1}^{k} c_{j}\left(x^{\prime \prime}, x^{\prime}\right) \otimes \delta^{(k-1)}\left(x_{m}\right)\right),
$$

where $c_{j}\left(x^{\prime \prime}, x^{\prime}\right) \in H^{s_{j}}\left(\mathbb{R}^{n+m-1}\right)$ are arbitrary functions, $s_{j}=s-\varkappa+j-1 / 2, j=1,2, \ldots, k$; lf is an arbitrary extension of $f$ to $H^{s-\alpha}\left(\mathbb{R}^{n+m}\right)$.

Proof. According to the theory of the multi-dimensional Riemann problem $[4,14]$, the function $Q^{-1} A_{=}^{-1} \widetilde{l f}$ can be represented as a sum of two terms (the so-called jump problem):

$$
Q^{-1} A_{=}^{-1} \widetilde{l f}=f_{+}+f_{-},
$$

where $f_{+} \in \tilde{H}\left(K_{n}\right), f_{-} \in \tilde{H}\left(\mathbb{R}^{m} \backslash K_{n}\right)$. Thus, we have

$$
Q^{-1} A_{\neq} \tilde{u}_{+}+Q^{-1} A_{=}^{-1} \tilde{u}_{-}=f_{+}+f_{-},
$$


or

In other words,

$$
Q^{-1} A_{\neq} \tilde{u}_{+}-f_{+}=f_{-}-Q^{-1} A_{=}^{-1} \tilde{u}_{-} .
$$

$$
A_{\neq} \tilde{u}_{+}-Q f_{+}=Q f_{-}-A_{=}^{-1} \tilde{u}_{-} .
$$

The left-hand side of this relation belongs to $\tilde{H}^{-k-\delta}\left(K_{n}\right)$ and the right-hand side belongs to $\tilde{H}^{-k-\delta}\left(\mathbb{R}^{n+m} \backslash K_{n}\right)$. Therefore,

$$
F^{-1}\left(A_{\neq} \tilde{u}_{+}-Q f_{+}\right)=F^{-1}\left(Q f_{-}-A_{=}^{-1} \tilde{u}_{-}\right),
$$

where the left-hand side belongs to $H^{-k-\delta}\left(K_{n}\right)$ and the right-hand side to $H^{-k-\delta}\left(\mathbb{R}^{n+m} \backslash K_{n}\right)$, which immediately implies that this is a generalized function with support in $\partial K_{n}$.

Denoting by $T_{a}$ the bijection $\mathbb{R}^{n+m} \rightarrow \mathbb{R}^{n+m}$ that transforms $\partial K_{n}$ into the hyperplane $x_{m}=0$ (more precisely, the transformation $\left.\left(x^{\prime \prime}, x^{\prime}, x_{m}\right) \rightarrow\left(x^{\prime \prime}, x^{\prime}, x_{m}-a\left|x^{\prime}\right|\right)\right)$, we see that the function

$$
T_{a} F^{-1}\left(A_{\neq} \tilde{u}_{+}-Q f_{+}\right)
$$

is supported on the hyperplane $t_{m}=0$ and belongs to $H^{-k-\delta}\left(\mathbb{R}^{n+m}\right)$. A generalized function of this type is a linear combination of the Dirac delta-function and its derivatives [18] and, in our case, has the form

$$
\sum_{j=0}^{k-1} c_{j}\left(t^{\prime \prime}, t^{\prime}\right) \otimes \delta^{(j)}\left(t_{m}\right)
$$

Simple calculations show that

$$
\begin{aligned}
&\left(F T_{a} u\right)(\xi)=\int_{\mathbb{R}^{m}} e^{-i x \cdot \zeta} \breve{u}\left(\xi^{\prime \prime}, x_{1}, \ldots, x_{m-1}, x_{m}-a\left|x^{\prime}\right|\right) d x \\
& \quad=\int_{\mathbb{R}^{m}} e^{-i y^{\prime} \xi^{\prime}} e^{-i\left(y_{m}+a\left|y^{\prime}\right|\right) \xi_{m}} \breve{u}\left(\xi^{\prime \prime}, y_{1}, \ldots, y_{m-1}, y_{m}\right) d y
\end{aligned}
$$

where $\breve{u}$ is the partial Fourier transform of $u$ in the first $n$ variables, the Jacobian of this transformation being equal to $1[7-9]$. Then

$$
\left(F T_{a} u\right)(\xi)=\int_{\mathbb{R}^{m-1}} e^{-i a\left|y^{\prime}\right| \xi_{m}} e^{-i y^{\prime} \cdot \xi^{\prime}} \hat{u}\left(\xi^{\prime \prime}, y_{1}, \ldots, y_{m-1}, \xi_{m}\right) d y^{\prime},
$$

where $\hat{u}$ is the Fourier transform of $u$ in the first $n$ and the last variables.

Since a pseudodifferential operator in $\mathbb{R}^{n+m}$ is defined by

$$
(A u)(x)=\int_{\mathbb{R}^{n+m}} e^{i x \cdot \xi} A(\xi) \tilde{u}(\xi) d \xi
$$

and the direct Fourier transform by

$$
\tilde{u}(\xi)=\int_{\mathbb{R}^{m}} e^{-i x \cdot \xi} u(x) d x
$$

it follows that we have obtained the relation

$$
\left(F T_{a} u\right)(\xi)=\int_{\mathbb{R}^{m-1}} e^{-i a\left|y^{\prime}\right| \xi_{m}} e^{-i y^{\prime} \cdot \xi^{\prime}} \hat{u}\left(\xi^{\prime \prime}, y_{1}, \ldots, y_{m-1}, \xi_{m}\right) d y .
$$

Denoting by $E_{a}\left(\xi^{\prime}, \xi_{m}\right)$ the $(m-1)$-dimensional Fourier transform $\left(y^{\prime} \rightarrow \xi^{\prime}\right.$ in the sense of distributions) of $e^{-i a\left|y^{\prime}\right| \xi_{m}}$, we can rewrite(4) as

$$
\left(F T_{a} u\right)(\xi)=\left(E_{a} * \tilde{u}\right)(\xi)
$$

where $*$ indicates convolution in the first $m-1$ variables and the operator of multiplication in the last variable $\xi_{m}$. 
Let (see also [7])

$$
V_{a}=F T_{a} F^{-1}
$$

Then, (5) can be rewritten as

$$
F T_{a}=V_{a} F
$$

Therefore, $V_{a}$ is a combination of convolution and the multiplicator with the kernel $E_{a}\left(\xi^{\prime}, \xi_{m}\right)$. This is a fairly simple operator, which is bounded in the Sobolev-Slobodetskii spaces $H^{s}\left(\mathbb{R}^{n+m}\right)$.

In order to obtain the statement of Theorem 2, it remains to perform some simple calculations with the operators $V_{a}, F$ and the distribution

$$
\sum_{j=0}^{k-1} c_{j}\left(t^{\prime \prime}, t^{\prime}\right) \otimes \delta^{(j)}\left(t_{m}\right)
$$

\section{Boundary-Value Problems with Local Boundary Conditions}

The expression (3) contains $k$ arbitrary functions. In order to obtain a unique solution, one needs additional conditions for the determination of these functions. As a rule, such conditions are formulated in terms of traces of some (pseudo)differential operators applied to the solution (3), but other types of additional conditions are also possible (see Sec. 5). One of the simplest cases of additional conditions is considered in [7-9]: for $k=1$, the classical Dirichlet or Neumann conditions. Here, we also limit ourselves with the Dirichlet condition, for the mere reason that the resulting constructions are too cumbersome.

Thus, let $k=1$ in (3). The expression becomes even simpler, if we take $f \equiv 0$ :

$$
\tilde{u}_{+}(\xi)=A_{\neq}^{-1}(\xi) \cdot V_{-a} \cdot F\left(c\left(x^{\prime \prime}, x^{\prime}\right) \otimes \delta\left(x_{m}\right)\right),
$$

where we have changed the notation: $c_{1}\left(x^{\prime \prime}, x^{\prime}\right) \equiv c\left(x^{\prime \prime}, x^{\prime}\right) \in H^{s-\varkappa+1 / 2}\left(\mathbb{R}^{n+m-1}\right)$.

Taking into account (6), we transform (7) as follows:

$$
u_{+}(x)=\left(F^{-1} \cdot A_{\neq}^{-1}(\xi) \cdot F\right) \cdot\left(F^{-1} \cdot V_{-a} \cdot F\right)\left(c\left(x^{\prime \prime}, x^{\prime}\right) \otimes \delta\left(x_{m}\right)\right) .
$$

Hence, using (6), we get

$$
u_{+}(x)=\left(A_{\neq}^{-1} \cdot T_{-a}\right)\left(c\left(x^{\prime \prime}, x^{\prime}\right) \otimes \delta\left(x_{m}\right)\right) .
$$

Remark 1. Here and above, $A_{\neq}^{-1}$ denotes the pseudodifferential operator with the symbol $A_{\neq}^{-1}(\xi)$, while $A_{\neq}^{-1}(\xi)$ stands for the function itself, as well as the operator of multiplication by this function; moreover, we will write $A_{\neq}^{-1} \tilde{v}$ instead of $A_{\neq}^{-1}(\xi) \tilde{v}(\xi)$.

Applying the operator $T_{a}$ to both sides of (8), we get

$$
\left(T_{a} u_{+}\right)(x)=\left(T_{a} \cdot A_{\neq}^{-1} \cdot T_{-a}\right)\left(c\left(x^{\prime \prime}, x^{\prime}\right) \otimes \delta\left(x_{m}\right)\right) .
$$

Suppose that the Dirichlet condition is prescribed on the boundary $\partial K_{n}$, i.e., the values of $u_{+}$on $\partial K_{n}$ are known. Therefore, the values of $\left(T_{a} u_{+}\right)(x)$ on the hyperplane $\left(x^{\prime \prime}, x^{\prime}, 0\right)$ are known. Denote this known function by $v\left(x^{\prime \prime}, x^{\prime}\right)$ :

$$
\left.\left(T_{a} u_{+}\right)(x)\right|_{x_{m}=0}=v\left(x^{\prime \prime}, x^{\prime}\right)
$$

Thereby, we come to the equation

$$
\left.\left(T_{a} \cdot A_{\neq}^{-1} \cdot T_{-a}\right)\left(c\left(x^{\prime \prime}, x^{\prime}\right) \otimes \delta\left(x_{m}\right)\right)\right|_{x_{m}=0}=v\left(x^{\prime \prime}, x^{\prime}\right)
$$

with the unknown function $c\left(x^{\prime \prime}, x^{\prime}\right)$. Applying the Fourier transformation to the last equation, we write it in the form

$$
\int_{-\infty}^{+\infty}\left(F \cdot T_{a} \cdot A_{\neq}^{-1} \cdot T_{-a}\right)\left(c\left(x^{\prime}, x^{\prime}\right) \otimes \delta\left(x_{m}\right)\right) d \xi_{m}=\tilde{v}\left(\xi^{\prime \prime}, \xi^{\prime}\right),
$$


or

$$
\int_{-\infty}^{+\infty}\left(F \cdot T_{a} \cdot F^{-1} \cdot F \cdot A_{\neq}^{-1} \cdot F^{-1} \cdot F \cdot T_{-a} \cdot F^{-1} \cdot F\right)\left(c\left(x^{\prime \prime}, x^{\prime}\right) \otimes \delta\left(x_{m}\right)\right) d \xi_{m}=\tilde{v}\left(\xi^{\prime \prime}, \xi^{\prime}\right),
$$

and after simplifications,

$$
\int_{-\infty}^{+\infty}\left(\left(V_{a} \cdot A_{\neq}^{-1}(\xi) \cdot V_{-a}\right) \tilde{c}\right)\left(\xi^{\prime \prime}, \xi^{\prime}, \xi_{m}\right) d \xi_{m}=\tilde{v}\left(\xi^{\prime \prime}, \xi^{\prime}\right)
$$

Equation (9) is an equivalent integral equation for the determination of the unknown function $c\left(x^{\prime \prime}, x^{\prime}\right) \in H^{s-\varkappa+1 / 2}\left(\mathbb{R}^{n+m-1}\right)$. Thus, the Dirichlet problem has a unique solution, if and only if equation (9) has a unique solution $c\left(x^{\prime \prime}, x^{\prime}\right) \in H^{s-\varkappa+1 / 2}\left(\mathbb{R}^{n+m-1}\right)$.

Theorem 3. For $\varkappa-s=1+\delta,|\delta|<1 / 2, f \equiv 0$, equation (1) has a unique solution in $H^{s}\left(K_{n}\right)$ satisfying the boundary condition $(*)$ with $v \in H^{s-\varkappa+1 / 2}\left(\mathbb{R}^{n+m-1}\right)$, if and only if the integral equation (9) has a unique solution in $H^{s-\varkappa+1 / 2}\left(\mathbb{R}^{n+m-1}\right)$.

\section{Boundary-Value Problems with Integral Boundary Conditions}

Here we consider another type of additional conditions that would allow us to uniquely determine the function $c\left(x^{\prime \prime}, x^{\prime}\right) \in H^{s-\varkappa+1 / 2}\left(\mathbb{R}^{n+m-1}\right)$, while staying in the framework of the special case of Sec. 4 and the expression of the general solution $(7)$.

It is convenient to write $(7)$ in the form

$$
\tilde{u}_{+}(\xi)=\left(\left(A_{\neq}^{-1}(\xi) \cdot V_{-a}\right) \tilde{c}\right)(\xi) .
$$

In order to determine the unknown function $c\left(x^{\prime \prime}, x^{\prime}\right)$, we suggest prescribing the integral of $u_{+}$over the boundary $\partial K_{n}$ (two-dimensional statements of the problem have been considered earlier in [13]). Thus, suppose that

$$
\int_{\partial K_{n}} u_{+}(y) d S_{y}
$$

is given. This is an integral of a function of $n+m$ variables over an $(n+m-1)$-dimensional surface. The result should be a function of a single variable. Let us represent this function of a single variable in another form with the help of the operator $T_{a}$ :

$$
\int_{\mathbb{R}^{n+m-1}}\left(T_{a} u_{+}\right)(x) d x^{\prime \prime} d x^{\prime}=v\left(x_{m}\right), \quad x=\left(x^{\prime \prime}, x^{\prime}, x_{m}\right) .
$$

From $\left(7^{\prime}\right)$, we get

$$
\left(T_{a} u_{+}\right)(x)=T_{a}\left(\left(F^{-1} \cdot A_{\neq}^{-1}(\xi) \cdot V_{-a}\right) \tilde{c}\right)(x), \quad x \in \mathbb{R}^{n+m} .
$$

In what follows, we need the following simple property of the Fourier transformation. Denote by $P^{\prime}$ the operator of restriction to the straight line $x_{m}$ in the $(n+m)$-dimensional space $\mathbb{R}^{n+m}$. It is easy to check that

$$
\left(P^{\prime} F u\right)\left(\xi_{m}\right) \equiv \tilde{u}\left(0,0, \xi_{m}\right)=\int_{\mathbb{R}^{n+m-1}} \hat{u}\left(x^{\prime \prime}, x^{\prime}, \xi_{m}\right) d x^{\prime \prime} d x^{\prime}
$$

Therefore,

$$
\left(F T_{a} u_{+}\right)\left(0,0, \xi_{m}\right)=\tilde{v}\left(\xi_{m}\right),
$$

and the last function is known. We can write

$$
\left(\left(F \cdot T_{a} \cdot F^{-1} \cdot A_{\neq}^{-1}(\xi) \cdot V_{-a}\right) \tilde{c}\right)(\xi)=\left(\left(V_{a} \cdot A_{\neq}^{-1}(\xi) \cdot V_{-a}\right) \tilde{c}\right)(\xi)
$$


and, after the restriction to the line, we get

$$
\left(\left(V_{a} \cdot A_{\neq}^{-1}(\xi) \cdot V_{-a}\right) \tilde{c}\right)\left(0,0, \xi_{m}\right)=\tilde{v}\left(\xi_{m}\right) .
$$

Relation (11) is an equivalent integral equation for the determination of $c\left(x^{\prime \prime}, x^{\prime}\right)$. Let us formulate the result obtained above.

Theorem 4. For $\varkappa-s=1+\delta,|\delta|<1 / 2, f \equiv 0$, equation (1) has a unique solution in $H^{s}\left(K_{n}\right)$ satisfying the boundary condition (10) with a given right-hand side, if and only if equation (11) has a unique solution in $H^{s-\varkappa+1 / 2}\left(\mathbb{R}^{n+m-1}\right)$.

Remark 2. Generally speaking, in order to ensure the unique solvability, one has to impose additional conditions on the integral equation (see [13]).

\section{Overdetermined Problems and Potentials}

In the remaining case, $\varkappa-s=k+\delta, k \in \mathbb{Z}, k<0,|\delta|<1 / 2$, we proceed as follows (see $[3-5,12,15]$ ). If the right-hand side of equation (1) belongs to $H_{0}^{s-\alpha}\left(K_{n}\right)$, then $f \in H_{0}^{\varkappa-\delta-\alpha}\left(K_{n}\right)$, since $s-\alpha=$ $\varkappa-n-\delta-\alpha \varkappa-\delta-\alpha$. By Theorem 1, there is a unique solution $w_{+} \in H^{\varkappa-\delta}\left(K_{n}\right)$ of (1) such that

$$
\tilde{w}_{+}(\xi)=A_{\neq}^{-1}(\xi) G_{n, m} A_{=}^{-1}(\xi) \widetilde{l f}(\xi) .
$$

The problem here is that (12) yields a solution of a wider class and there is still a certain arbitrariness in the choice of the solution from the desired class $H^{s}\left(K_{n}\right)$.

As in Secs. 4 and 5 above, we "reduce" equation (12) to the case of a half-space and use the results obtained in that case [3]. From [3], we borrow some facts connected with the theory of one-dimensional singular integral equations and the classical Riemann problem $[1,2]$.

We introduce the following operators initially defined on the Schwartz space $S\left(\mathbb{R}^{n+m}\right)$ consisting of infinitely differentiable functions rapidly decaying at infinity:

$$
\left(\Pi_{ \pm} \tilde{u}\right)\left(\xi^{\prime \prime}, \xi^{\prime}, \xi_{m}\right)= \pm \frac{i}{2 \pi} \lim _{\tau \rightarrow 0+} \int_{-\infty}^{+\infty} \frac{\tilde{u}\left(\xi^{\prime \prime}, \xi^{\prime}, \eta_{m}\right) d \eta_{m}}{\xi_{m} \pm i \tau-\eta_{m}}, \quad\left(\Pi^{\prime} \tilde{u}\right)\left(\xi^{\prime \prime}, \xi^{\prime}\right)=\int_{-\infty}^{+\infty} \tilde{u}\left(\xi^{\prime \prime}, \xi^{\prime}, \xi_{m}\right) d \xi_{m},
$$

and let $\Lambda_{ \pm}$be the pseudodifferential operator with the symbol $\Lambda_{ \pm}\left(\xi^{\prime}, \xi_{m}\right)=\xi_{m} \pm i\left|\xi^{\prime}\right| \pm i$. Then, for any $\tilde{u} \in \tilde{H}^{k+\delta}\left(\mathbb{R}^{n+m}\right), k \in \mathbf{N},|\delta|<1 / 2$, we have (see [3])

$$
\left(\Pi_{+} \tilde{u}\right)\left(\xi^{\prime \prime}, \xi^{\prime}, \xi_{m}\right)=\sum_{j=1}^{k} \frac{i\left(\Pi^{\prime} \Lambda_{+}^{j-1} \tilde{u}\right)\left(\xi^{\prime \prime}, \xi^{\prime}\right)}{\Lambda_{+}^{j}\left(\xi^{\prime}, \xi_{m}\right)}+\frac{1}{\Lambda_{+}^{k}\left(\xi^{\prime}, \xi_{m}\right)}\left(\Pi_{+} \Lambda_{+}^{k} \tilde{u}\right)\left(\xi^{\prime \prime}, \xi^{\prime}, \xi_{m}\right) .
$$

Now, we rewrite (12) in the form

$$
F_{\xi \rightarrow x}^{-1}\left(A_{\neq}(\xi) \tilde{w}_{+}(\xi)\right)=F_{\xi \rightarrow x}^{-1}\left(G_{n, m} A_{=}^{-1}(\xi) \widetilde{l f}(\xi)\right),
$$

which implies that

$$
\left(A_{\neq} * w_{+}\right)(x)=\chi_{\mathbb{R}^{n} \times C_{+}^{a}}(x) \cdot\left(A_{=}^{-1} *(l f)\right)(x),
$$

where $\chi_{\mathbb{R}^{n} \times C_{+}^{a}}$ is the characteristic function of the set $\mathbb{R}^{n} \times C_{+}^{a}$.

Let us apply the operator $T_{a}$ to both sides of the last relation and pass to the Fourier transforms. Note that

Therefore,

$$
T_{a}\left(\chi_{\mathbb{R}^{n} \times C_{+}^{a}}(x) \cdot\left(A_{=}^{-1} *(l f)\right)(x)\right)=\chi_{\mathbb{R}^{n} \times \mathbb{R}_{+}^{m}}(x) \cdot T_{a}\left(A_{=}^{-1} *(l f)\right) .
$$

and we further have

$$
\left(F T_{a}\right)\left(A_{\neq} * w_{+}\right)(\xi)=\Pi_{+} V_{a}\left(A_{=}^{-1}(\xi) \widetilde{l f}(\xi)\right),
$$

$$
\tilde{w}_{+}(\xi)=A_{\neq}^{-1}(\xi) V_{-a} \Pi_{+} V_{a}\left(A_{=}^{-1}(\xi) \widetilde{l f}(\xi)\right) .
$$


Using $(*)$ in the last relation, we obtain the desired representation

$$
\tilde{w}_{+}(\xi)=\sum_{j=1}^{k}\left[\left(A_{\neq}^{-1} \cdot V_{-a} \cdot \Lambda_{+}^{-j} \cdot V_{a}\right) \tilde{v}_{j}\right]\left(\xi^{\prime \prime}, \xi^{\prime}, \xi_{m}\right)+\tilde{u}_{+}(\xi),
$$

where [3]

$$
\tilde{v}_{j}\left(\xi^{\prime \prime}, \xi^{\prime}\right)=i\left(\Pi^{\prime} \Lambda_{+}^{j-1} \cdot A_{=}^{-1} \cdot \widetilde{l f}\right)\left(\xi^{\prime \prime}, \xi^{\prime}\right), \quad \tilde{u}_{+}(\xi)=A_{\neq}^{-1}(\xi) V_{-a} \Lambda_{+}^{-k}\left(\Pi_{+} V_{a} \Lambda_{+}^{k} A_{=}^{-1} \cdot \tilde{l f}\right)\left(\xi^{\prime \prime}, \xi^{\prime}, \xi_{m}\right) .
$$

Let us single out the terms

$$
V_{-a} \Lambda_{+}^{-j} V_{a} \tilde{v}_{j} \equiv \tilde{u}_{j}
$$

and rewrite our representation in the form

$$
\tilde{w}_{+}(\xi)=\sum_{j=1}^{k}\left(A_{\neq}^{-1} \cdot \tilde{u}_{j}\right)\left(\xi^{\prime \prime}, \xi^{\prime}, \xi_{m}\right)+\tilde{u}_{+}(\xi) .
$$

The terms in (14) play the role of potentials.

Theorem 5. For $\varkappa-s=k+\delta, k \in \mathbf{Z}, k<0,|\delta|<1 / 2$, equation (1) has a solution in $H^{s}\left(K_{n}\right)$, if and only if the right-hand side $f$ satisfies the additional conditions

$$
\tilde{u}_{j}=0, \quad j=1, \ldots,|k| \text {. }
$$

\section{Conclusion}

Obviously, we have not considered here all possible combinations of singularities that can be examined by our methods. In particular, one of the most interesting problems, in our opinion, is to investigate singularities described by the product of two cones: one cone $C_{+}^{a} \subset \mathbb{R}^{n}$ with respect to one part of the variables and another, $C_{+}^{b} \subset \mathbb{R}^{m}$, with respect to the other part. The existence of solutions of equation (1) and the corresponding boundary-value problems can be examined in the space $H^{s}\left(C_{+}^{a} \times C_{+}^{b}\right) \subset H^{s}\left(\mathbb{R}^{n+m}\right)$. The author hopes to deal with these situations in further publications.

\section{REFERENCES}

1. F. D. Gakhov, Boundary-Value Problems [in Russian], Nauka, Moscow (1977).

2. N. I. Muskhelishvili, Singular Integral Equations [in Russian], Nauka, Moscow (1968).

3. G. I. Eskin, Boundary-Value Problems for Elliptic Pseudodifferential Equations [in Russian], Nauka, Moscow (1973).

4. V. B. Vasilyev, Multiplicators of Fourier Integrals. Pseudodifferential Equations. Wave Factorization. Boundary-Value Problems [in Russian], KomKniga, Moscow (2010).

5. V. B. Vasilyev, Wave Factorization of Elliptic Symbols: Theory and Applications. Introduction to Boundary-Value Problems in Non-Smooth Domains, Kluwer Academic, Dordrecht (2000).

6. V. B. Vasilyev, "Pseudodofferential equations in cones with conjugation points at the boundary," Differ. Uravn., 51, No. 9, 1123-135 (2015).

7. V. B. Vasilyev, "On the Dirichlet and Neumann problems in multi-dimensional cone," Math. Bohem., 139, No. 2, 333-340 (2014).

8. V. B. Vasilyev, Potentials for Elliptic Boundary-Value Problems in Cones, arXiv:1409.4492.

9. V. B. Vasilyev, "On certain elliptic problems for pseudo-differential equations in a polyhedral cone," Adv. Dyn. Syst. Appl., 9, No. 2, 227-237 (2014).

10. V. B. Vasilyev, "Pseudodifferential equations, singular integrals and distributions," Prikl. Mat. Mat. Fiz., 1, No. 1, 3-16 (2015).

11. V. B. Vasilyev, "New constructions in the theory of elliptic boundary-value problems," in: C. Constanda and A. Kirsch, eds., Integral Methods in Science and Engineering. Proc. IMSE Conference, Karlsruhe, Germany, 2014, Birkhäuser, Basel (2015), pp. 573-584. 
12. V. B. Vasilyev, "Potential like operators in the theory of boundary-value problems in nonsmooth domains," Azerb. J. Math., 2, No. 2, 117-128 (2012).

13. V. B. Vasilyev, "Some new boundary-value problems in nonsmooth domains," in: Itogi Nauki Tekh. Ser. Sovr. Mat. Prilozh., 67, 101-106 (2010).

14. V. B. Vasilyev, "Regularization of multi-dimensional singular integral equations in nonsmooth domains," Tr. Mosk. Mat. Obshch., 59, 73-105 (1998).

15. V. B. Vasilyev, "Some classes of pseudodifferential equations in cones," Dokl. RAN, 355, No. 1, 14-17 (1997).

16. S. Bochner and W. T. Martin, Several Complex Variables, Princeton Univ. Press, Princeton (1948).

17. V. S. Vladimirov, Methods of the Theory of Several Complex Variables [in Russian], Nauka, Moscow (1964).

18. V. S. Vladimirov, Generalized Functions in Mathematical Physics [in Russian], Nauka, Moscow (1979).

V. B. Vasilyev

Lipetsk State Technical University, Lipetsk, Russia

E-mail: vladimir.b.vasilyev@gmail.com 